\title{
Saturation des lignes ferroviaires et choix d'investissement, le cas de la Ligne grande vitesse Paris-Lyon
}

\section{Railway saturation and investment choice, the case of the High speed line Paris-Lyon}

\author{
Florent Laroche \\ Reçu le 4 novembre 2013 ; accepté le 8 juillet 2014 \\ (C) IFSTTAR et Éditions NecPlus 2015
}

\begin{abstract}
Résumé Ce papier propose une méthode pour conceptualiser la saturation d'une infrastructure ferroviaire. Elle est appliquée au cas de la Ligne à Grande Vitesse Paris-Lyon et permet de tester et comparer plusieurs pistes de désaturation. Cette méthode peut être considérée comme un outil de décision au service des politiques publiques pour guider le choix en investissements.
\end{abstract}

Mots clés prospective, saturation, ferroviaire, LGV, investissement, innovation

\begin{abstract}
This paper proposes a method to understand saturation in the railways. It will be applied to the case of Lyon-Paris High-Speed Line. The method presented enables to test and compare different solutions to cope with saturation. It can be considered as both a tool for decision-makers and a support to investment choices.
\end{abstract}

Keywords Forecast, Saturation, Railway, HSL, Investment, Innovation

\section{Introduction}

Le Grenelle de l'environnement (2007) a eu pour résultat de définir une politique de transport durable en France en donnant priorité au report modal des trafics voyageurs et marchandises de la route vers le rail. Dans cette perspective un Schéma national des infrastructures de transport (SNIT) a été établi, en 2011 [1], en faveur d'un doublement du réseau de Lignes à grande vitesse (LGV) d'ici à 2020. Néanmoins, un an seulement après son officialisation, une commission, « mobilité $21 »$, a été créée pour repenser le schéma de développement du réseau LGV en accord

Florent Laroche $(\bowtie)$

Université de Lyon, Laboratoire d'Économie des Transports

14 avenue Berthelot, 69363 Lyon, France

e-mail : florent.Laroche@1et.ish-lyon.cnrs.fr avec les besoins et les moyens financiers disponibles sous contrainte de la raréfaction des fonds publics. La révision du SNIT pose deux questions cruciales pour l'avenir du réseau LGV français : quelle pertinence dans la poursuite de son extension [2,3] ? Quelle gestion de sa maturité et du risque de saturation pour ses lignes les plus anciennes [4]?

Ce papier discute le risque de saturation des LGV selon une approche socio-économique de la capacité ferroviaire. Il repose sur une modélisation simplifiée de la capacité et propose de mener une réflexion sur les différents leviers existants pour ajuster l'offre en fonction de la demande. L'objectif est d'identifier ces leviers et de définir des pistes possibles d'investissement pour limiter la saturation.

Une revue de littérature montre que si de nombreuses méthodes génériques existent pour évaluer le taux d'utilisation d'une infrastructure routière, peu ont été développées pour le ferroviaire. Le ferroviaire se distingue par une plus grande variété et complexité des systèmes [5] et par un plus grand nombre de leviers pour maîtriser la capacité (activité planifiée et centralisée). En conséquence, le choix a été fait de développer une méthode propre aux LGV françaises. Elles présentent notamment la particularité d'avoir des flux homogènes à la fois en termes de vitesse et de services. Côté demande, les récents travaux de prospective en trafic [6,7] s'accordent sur deux points. En premier lieu, la demande globale en transport devrait continuer de s'accroître à l'horizon 2050, à moins de considérer une période longue de décroissance, la demande en transport étant positivement corrélée à l'évolution du PIB [8]. En second lieu, l'évolution modale devrait être largement favorable au transport ferroviaire sous l'effet de deux paramètres : une élasticité vitesse/PIB positive et des mesures de plus en plus contraignantes en termes de politiques publiques pour la diminution de la vitesse routière et en faveur de la grande vitesse ferroviaire. Ainsi, la demande devrait s'accroître au minimum de $50 \%$ d'ici à 2050 , d'où le questionnement suivant : dans quelle mesure l'infrastructure pourra-t-elle supporter l'évolution du trafic 
et de quels leviers dispose-t-on pour limiter l'effet de saturation? L'enjeu est d'autant plus sensible dans le cas de la LGV Paris-Lyon qu'il existe un projet de doublement de la voie en débat (Paris-Orléans-Clermont-Ferrand-Lyon, POCL) évalué à près de 14 milliards d'euros [9].

Dans un premier temps, le papier revient sur la notion de capacité d'une infrastructure selon une double approche technique et économique. Puis, la méthode et les hypothèses retenues sont présentées et illustrées par le cas de la LGV Lyon-Paris. Enfin, les résultats sont analysés sous la forme d'une évaluation socio-économique des différentes pistes d'adaptation possibles de l'outil de production.

\section{Appréhender la capacité ferroviaire comme un système complexe}

Dans le cas du ferroviaire, deux approches prévalent pour conceptualiser la capacité : socio-économique et technique. Considérant le système ferroviaire comme un système complexe [10], ce premier point rappelle les enjeux des deux approches puis propose de les faire converger dans une représentation d'ensemble de la capacité ferroviaire.

\section{Deux approches distinctes}

On peut distinguer l'approche traditionnelle de l'ingénierie pour une évaluation technique de la capacité de l'approche économique plus récente concernant la répartition de cette capacité et sa tarification.

La littérature questionnant la notion de capacité, pour une infrastructure ferroviaire (voie unique et double), est déjà ancienne chez les ingénieurs [11]. La recherche des déterminants techniques de la capacité et de son optimisation a été le principal fil conducteur des études menées depuis les années 1950. Pourtant, il n'existe pas de modèle standard comme pour le routier [5]. Le système est dans la plupart des cas considéré comme intégré et la réflexion est menée selon les particularités du réseau [12]. De cette diversité, quelques orientations communes de recherche sont identifiables à travers la littérature. Le lien entre capacité et robustesse des horaires apparaît comme une problématique clef du système. Elle passe par la maîtrise de l'hétérogénéité des trafics $[13,14]$ et la gestion des retards $[15,16,17]$. Par ailleurs, la notion de capacité pour une ligne est souvent considérée comme complexe à définir et varie selon les critères retenus, tout comme le phénomène de saturation qu'il faut considérer avec prudence $[18,19,20]$. Enfin, de nombreuses solutions techniques existent pour résorber les problèmes de capacité à condition de moderniser les outils et méthodes de production $[21,22,23,24]$. De manière générale, il est intéressant de noter que ces recherches ont souvent été réalisées pour le compte de compagnies ferroviaires ce qui a contribué à les rendre peu lisibles par les acteurs publics responsables des choix d'investissements. Le champ technique semble en effet souvent considéré comme un domaine réservé et les acteurs publics sont convoqués sur les problèmes de capacité lorsque l'opérateur juge l'ensemble des solutions techniques épuisées pour résoudre le problème et propose la création d'une nouvelle infrastructure.

Les économistes se sont emparés plus tardivement de cette problématique suite à la déréglementation du système ferroviaire Suédois en 1988. On s'intéresse alors à l'interaction entre offre et demande sous contrainte de capacité du réseau. L'étude des déterminants de la capacité devient celle de la valeur d'un sillon selon la charge de l'infrastructure [25]. Sous l'effet de la séparation verticale entre gestionnaire d'infrastructure et opérateur de réseau, le système ne s'autorégule plus par lui-même et des asymétries dans l'information apparaissent [26]. Selon cette problématique, la littérature s'articule autour de deux axes de recherche : quel mode d'allocation de la capacité pour assurer la non-discrimination dans l'accès au réseau $[27,28,29]$ et quelle évaluation de la rareté du sillon dans un souci d'optimisation de l'utilisation du réseau $[30,31,4]$ ?

Ce papier s'inscrit à la croisée des deux approches. La démarche proposée est propre à l'approche économique (logique de demande et valorisation du réseau) et s'inspire des travaux techniques réalisés pour représenter la contrainte dans l'offre et identifier les éléments clefs susceptibles de faire évoluer cette contrainte selon les impératifs de la demande.

\section{Concilier les deux approches dans une analyse de la capacité}

Le système ferroviaire se caractérise par une interaction complexe entre ingénierie et logique économique. La capacité est définie en cohérence avec ces deux logiques. L'aspect technique comprend quatre composantes clefs : infrastructure, exploitation, gestion des trafics, matériel roulant. L'aspect économique contient la composante tarification de l'infrastructure et du service. Elle dépend du comportement des acteurs et permet de moduler la demande en transport (théorie des surplus). Par conséquent, ces composantes sont à la fois des facteurs de contrainte pour l'offre mais aussi des variables d'ajustement selon le niveau de demande. Leur dynamique dépend pour l'essentiel des acteurs concernés et de leurs choix, le système ferroviaire étant par nature une activité planifiée.

L'infrastructure est a priori neutre mais peut être composée de spécificités selon les services envisagés 


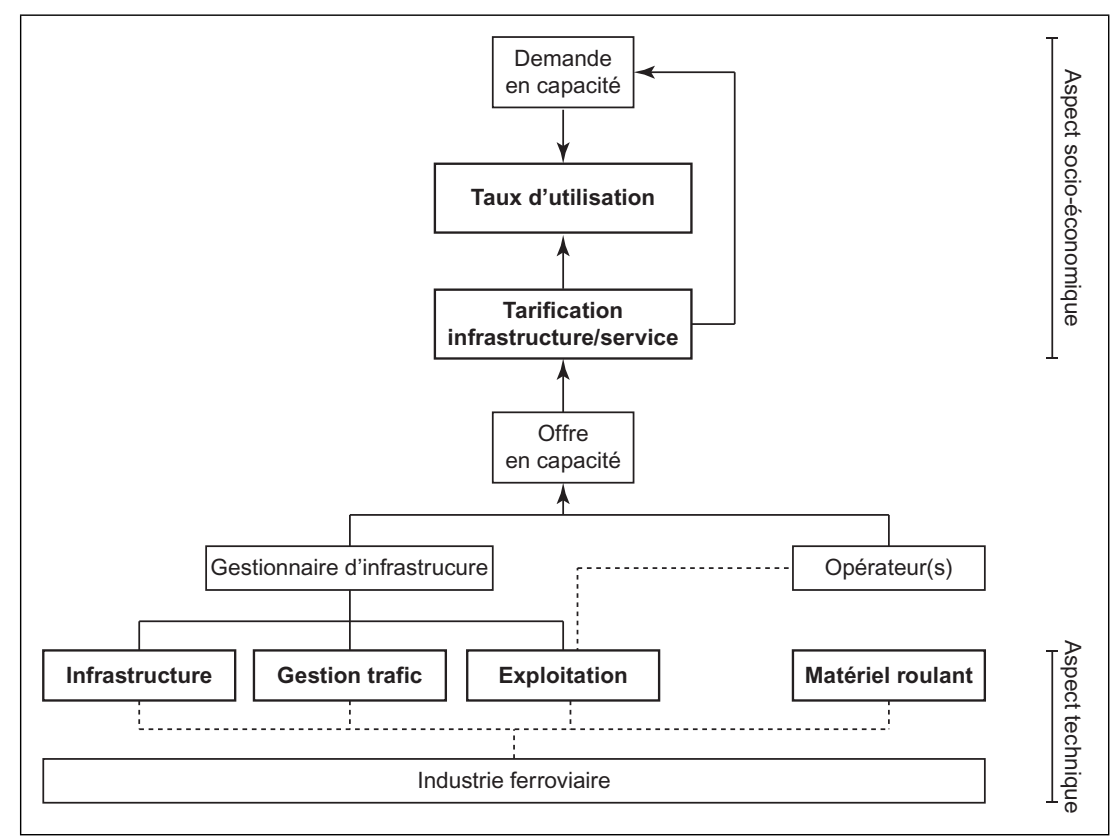

Fig. 1 Composantes essentielles de la capacité ferroviaire (source : auteur)

comme c'est le cas pour les LGV qui n'acceptent que les services de type TGV. Elles se distinguent des lignes classiques qui accueillent aussi bien des trains voyageurs longue distance que des trains de fret ou de voyageurs courte distance (forte hétérogénéité). Cette diversité des profils de ligne peut entraîner des variations importantes en termes de capacité d'une ligne à l'autre. L'architecture des LGV permet une vitesse plus élevée que sur ligne classique mais n'offre pas forcément une plus grande capacité théorique du fait de la vitesse et de ses contraintes [32,33]. Dans ce cas, la capacité dépend de la composition des trafics, la LGV ayant une homogénéité quasi parfaite des trafics et du matériel roulant (un seul type de service ferroviaire) son optimisation peut être simplifiée par rapport à une ligne classique [32,5].

L'exploitation est une composante essentielle de la capacité. Elle détermine la capacité maximale d'une ligne ferroviaire selon le système d'exploitation adopté (système de contrôle-commande). Le système ferroviaire étant un mode de transport guidé, cette composante concerne à la fois le gestionnaire d'infrastructure, responsable de l'infrastructure et l'opérateur de service dont le système de signalisation embarqué par le train doit correspondre au système sur voie.

La troisième composante, la gestion du trafic, a essentiellement trait à la capacité du régulateur de trafic à gérer les flux en temps réel. Il s'inscrit en «chef d'orchestre » de la capacité à l'instant « $\mathrm{t}$ » et ses interactions avec les autres composantes sont multiples. Sa performance dépend à la fois du système de transmission des informations, de la souplesse du système d'exploitation, de la configuration de l'infrastructure (voie d'évitement, etc.) mais aussi de la gestion des priorités et de la capacité du régulateur à reconfigurer l'itinéraire d'un train en cas de perturbation.

La quatrième composante concerne le matériel roulant et sa capacité intrinsèque. Seule la capacité d'un train en voyageurs est prise en compte (ou rame dans le cas des TGV). L'hypothèse est faite que les caractéristiques techniques du matériel roulant sont intégrées dans les parties exploitation et infrastructure (temps de freinage, vitesse, masse, etc.). Cette composante permet de représenter les choix des opérateurs et de mesurer leur impact sur l'offre générale en transport.

Enfin, la cinquième et dernière composante définit la valorisation économique de la capacité ferroviaire. Elle est le résultat des choix réalisés par les acteurs selon les composantes techniques, sous contrainte de la demande, et résulte de relations complexes. L'opérateur fait office d'intermédiaire entre le prix défini par le gestionnaire pour l'utilisation de l'infrastructure (prix de gros) et le coût supporté par les clients (prix de détail). Ce facteur prix peut constituer une variable d'adaptation de la demande à la capacité disponible par effet de rareté.

La méthode s'appuie sur ces cinq composantes considérées comme déterminants essentiels de la capacité. Il est à noter ici que la répartition des composantes selon les acteurs s'inspire de la définition européenne (Directive 91/440/CE) avec un gestionnaire d'infrastructure unifié regroupant la gestion de l'infrastructure, des trafics et de l'exploitation du système. L'opérateur de service a pour seule mission d'assurer le transport de voyageurs ou de marchandises. 
On trouve en amont de la chaîne les industries ferroviaires qui fournissent les matériels et composants essentiels au système.

\section{Proposition d'une méthode pour évaluer la saturation des LGV}

Cette partie propose une formalisation de la notion de capacité en vue de sa modélisation dans le cas d'une LGV. La capacité de l'infrastructure peut être définie basiquement comme le «nombre de train pouvant circuler sur une section donnée, en un temps donné » [19].

\section{Formalisation de la notion de capacité}

L'équation de capacité peut être formalisée à partir du schéma suivant. Il met en évidence les différentes interactions du système et les différents seuils de saturation.

L'analyse repose sur le taux d'utilisation d'une ligne ferroviaire. Ce taux mesure le rapport entre la demande (D) exprimée en voyageurs et la capacité (C) d'une infrastructure exprimée en sillons (équivalent trains) sur un temps donné.

La littérature distingue deux types de capacité. La capacité «théorique » $(\mathrm{Ct})$ ou « absolue » équivaut au nombre maximal de sillons défini sur une ligne en un temps donné $[18,19]$. Elle se distingue de la capacité «pratique » ou « commerciale » $(\mathrm{Cc})$ qui équivaut au nombre de sillons disponibles pour la commercialisation $[18,20]$.
L'écart entre les deux types de capacité renvoie à la notion clef de « souplesse » du système (ou de « résilience ») pour une activité planifiée. L'écart peut être formalisé sous la forme d'un coefficient de «souplesse » appliqué à la capacité théorique pour déterminer la capacité pratique. Il traduit essentiellement la capacité mise en réserve pour prévenir les situations perturbées et limiter les retards en cascade [34]. L'importance de cette réserve est déterminée par les composantes «exploitation » (performance du système d'exploitation) et « gestion de trafic » (capacité à traiter les perturbations). Par ailleurs, les études économétriques sur les déterminants des retards font état d'une relation non linéaire entre la densité de trafic et le risque de retard [35,36,4]. Au-delà d'un certain seuil, le risque de retard moyen s'accroît de manière exponentielle. L'analyse économétrique montre qu'en général le seuil est fixé de telle sorte que le risque de retard moyen ne dépasse pas 10 minutes. Cette valeur ne semble pas être un objectif constitué en soi mais plutôt une valeur observée de fait $[35,36,4]$. Ce seuil dépend directement du choix du gestionnaire d'infrastructure, de sa sensibilité au risque et de la pression exercée par la demande.

En conséquence, définir le coefficient de «souplesse » d'un réseau, c'est évaluer pour partie sa performance technique mais aussi économique.

Pour finir, Brunel et al. [4] distingue la capacité intravoyageurs (dans les trains) et la capacité inter-voyageurs (entre les trains). Cette capacité dépend directement des choix en matière de service réalisés par les opérateurs.

Considérons maintenant le concept de saturation. Sa définition repose sur la lecture du taux d'utilisation selon

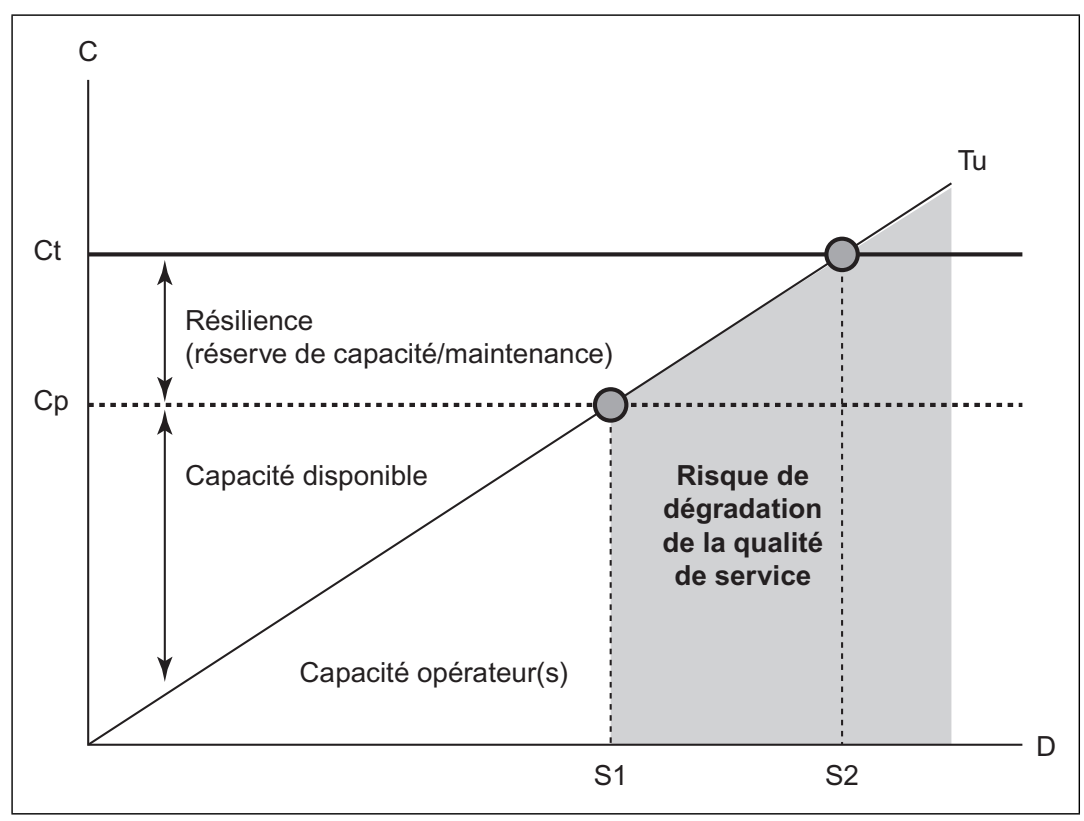

Fig. 2 Taux d'utilisation et notion de capacité (source : auteur) 
la capacité théorique de l'infrastructure. Deux seuils sont identifiés dans le schéma. Le seuil S2 dépend de la capacité théorique. Au-delà de ce seuil, l'infrastructure est déclarée saturée (taux d'utilisation supérieur à $100 \%$ ). Le seuil S1 dépend de la capacité «commerciale». Il peut être amené à évoluer à la hausse ou à la baisse selon l'arbitrage réalisé par le gestionnaire d'infrastructure entre densité de trafic et risque de retard.

Il est intéressant à noter que ces deux seuils dépendent de logiques différentes. Pour S2, il est possible de parler d'une capacité «structurelle » dont l'évolution dépend d'investissements lourds en infrastructure ou en exploitation sur le moyen et long terme tandis que $\mathrm{S} 1$ fait plutôt référence à une capacité « du quotidien » qui dépend des choix et de la performance des acteurs.

Par conséquent, les concepts de capacité et de saturation sont variables. La modélisation dans le point suivant est une tentative de prise en compte de ces interactions complexes.

\section{Formalisation de la méthode}

La méthode est calibrée pour le cas d'une LGV dont les trafics sont considérés homogènes en termes de vitesse et de service. Il s'inscrit ici dans une logique de tronçon avec une entrée et une sortie. L'objectif est de confronter la demande réelle et projetée à l'offre pour évaluer les besoins en capacité. Par conséquent, l'indicateur recherché est le taux d'utilisation ( $\mathrm{Tu}$ ). Il est calculé selon le rapport entre la demande en voyageurs et l'offre en capacité exprimée trains (équivalent voyageurs).

La demande (D) est définie selon le nombre de voyageurs (V) pondéré par le coefficient de concentration $(\phi)$. Ce coefficient permet de raisonner en heure de pointe en évaluant le différentiel moyen de concentration de la demande entre l'heure de pointe et l'heure creuse.

$$
\mathrm{D}=\mathrm{V}^{*} \phi
$$

L'offre en capacité est définie selon le nombre de places disponibles par train $(\mathrm{T})$ et le nombre de sillons (équivalent trains) disponibles par heure (S) sur la ligne (capacité commerciale).

Test obtenu en pondérant la capacité par rame $(\mathrm{Cr})$ par le taux de remplissage $(\theta)$ qui révèle sa véritable utilité sociale et le taux d'unité multiple $(\mu)$. Le taux d'unité multiple correspond à la composition des TGV qui peut être constituée d'une ou deux rames.

$$
\mathrm{T}=\left(\mathrm{Cr}^{*} \theta\right)^{*} \mu
$$

$\mathrm{S}$ est obtenu en pondérant la capacité théorique $(\mathrm{Ct}) \mathrm{de}$ l'infrastructure par le coefficient de souplesse $(\kappa)$.

$$
\mathrm{S}=\mathrm{Ct}^{*} \kappa
$$

L'équation générale s'écrit de la manière suivante, où $\mathrm{h}$ exprime l'amplitude horaire de mise à disposition de l'infrastructure pour les opérations commerciales sur une journée.

$$
\mathrm{Tu}=\left(\mathrm{D}^{*} \phi\right) /\left[\left(\left(\mathrm{Cr}^{*} \theta\right)^{*} \mu\right)^{*}\left(\left(\mathrm{Ct}^{*} \kappa\right)^{*} \mathrm{~h}\right)\right]
$$

Cette équation permet de tester la sensibilité du taux d'utilisation par rapport à l'évolution de la demande dans le temps selon différents paramètres. On peut dans ce cas évaluer l'horizon de saturation d'une infrastructure.

\section{Application sur le cas de la LGV Paris-lyon}

Cette section propose d'appliquer la méthode au cas de la ligne LGV Paris-Lyon selon deux objectifs : évaluer l'horizon de saturation de la ligne et identifier les différents choix possibles en matière d'investissement pour repousser cet horizon.

Dans un premier temps, les hypothèses de demande et d'offre sont présentées puis les résultats sont analysés sous la forme de scénarios possibles d'adaptation de l'offre à la demande.

\section{Hypothèses de demande : test de sensibilité}

On utilise les perspectives de trafic proposées par RFF dans l'étude réalisée sur le POCL [6] pour définir la demande à l'horizon 2050. La demande en voyageurs observée en 2008 est de 38 millions de voyageurs sur la LGV Paris-Lyon. Elle devrait être de 83 millions en $2050(+115 \%)$ et mener à la saturation de la ligne à horizon 2025. Cette croissance s'explique par un rapport demande en vitesse/PIB positif, bien que dégradé sous l'effet de la crise économique jusqu'en 2025, et par une augmentation de la taille du réseau LGV du fait des développements attendus pour l'axe Sud-Est par le SNIT [1].

Le scénario « corrigé » propose de tester la sensibilité de la demande aux deux variables clefs du scénario proposé par RFF : croissance du PIB et effet de réseau. En premier lieu, les hypothèses de croissance sont volontairement réduites selon un scénario de marasme économique persistant de type « économie japonaise ». En second lieu, l'élasticité vitesse/PIB est maintenue dégradée alors qu'elle devrait être, selon la littérature, proche de $1[8,37]$. On pose l'hypothèse qu'un découplage pourrait être progressivement obtenu sous l'effet dans un premier temps de la crise économique puis rendu durable par le développement de nouvelles pratiques liées aux technologies numériques 


\begin{tabular}{|lcc|}
\hline \multicolumn{2}{|c|}{ Tableau 1 Détail des hypothèses retenues pour la demande (source : auteur selon [6]) } \\
\hline & Hypothèses PIB & Hypothèses Réseau \\
\hline Scénario RFF initial & $+1,45 \% /$ an pour 2009-2025 & Réalisation des projets du SNIT pour l'axe Sud-Est \\
(2011) SI & Élasticité vitesse/PIB : $0,6+1,8 \% /$ an pour 2026 - & en $2025:$ LGV Lyon-Turin, LGV PACA, \\
& 2050 & Contournement Nîmes-Montpellier, LGV \\
& Élasticité vitesse/PIB : 0,9 & Rhin-Rhône (branche Ouest et Sud) \\
Scénario corrigé (2013) & $+1,05 \% /$ an pour $2009-2025$ & Réalisation des projets pour l'axe Sud-Est : LGV \\
SC & Élasticité vitesse/PIB : $0,6+1,5 \% /$ an pour 2026 - & Lyon-Turin, LGV PACA, Contournement \\
& 2050 & Nîmes-Montpellier, LGV Rhin-Rhône (branche \\
& Élasticité vitesse/PIB : 0,6 & Ouest et Sud)
\end{tabular}

(téléconférence, etc.) ou de nouvelles habitudes de mobilité (covoiturage, etc.). Enfin, la sensibilité de la demande à l'effet réseau est testée en posant l'hypothèse de l'abandon de deux projets majeurs pour l'axe Sud-Est inscrits dans le SNIT, la LGV Lyon-Turin et la LGV PACA.

On obtient donc deux scénarios de demande. Le scénario initial (SI), tel quel proposé par RFF, et le scénario corrigé (SC).

\section{Hypothèses d'offre et identification des pistes d'adaptation de l'offre à la demande}

Comme la demande, l'offre est considérée de manière dynamique. Face au risque de saturation, plusieurs pistes d'accroissement de la capacité peuvent être explorées. Il est proposé dans le tableau suivant de raisonner sur la base de la situation en 2008 et de modifier certains paramètres selon les types d'évolution de la capacité envisagés. Ces types d'évolution s'inspirent des composantes clefs du système ferroviaire présentées dans la première section et représentent autant de leviers possibles pour adapter l'offre à la demande. Ces pistes (ou scénarios) sont au nombre de cinq : l'infrastructure (S1), le matériel roulant (S2), la gestion de trafic (S3), l'exploitation (S4) et la tarification (S5). L'horizon d'évolution est fixé à 2025 et les solutions proposées sont toutes considérées comme possibles à mettre en œuvre.

Seules les valeurs retenues pour la situation de référence sont présentées ici. Les valeurs pour les autres scénarios seront présentées dans la section suivante en même temps que les résultats.

La situation en 2008, selon l'analyse de l'étude menée par RFF [6] sur le projet POCL, est la suivante. La capacité théorique moyenne des rames en circulation sur la ligne Paris-Lyon est de 450 voyageurs. Le résultat est obtenu à partir d'une moyenne pondérée par les rames duplex à 512 places et les rames réseau encore en circulation vers la Suisse dont la capacité est limitée à 377 places. Le taux d'unité multiple observé est de 1,3 en heure de pointe et le taux de remplissage moyen de $80 \%$. L'opérabilité du réseau est de 18 h ( 5 h 30-23 h 30). La capacité théorique est de 16 trains par heure selon le système de signalisation

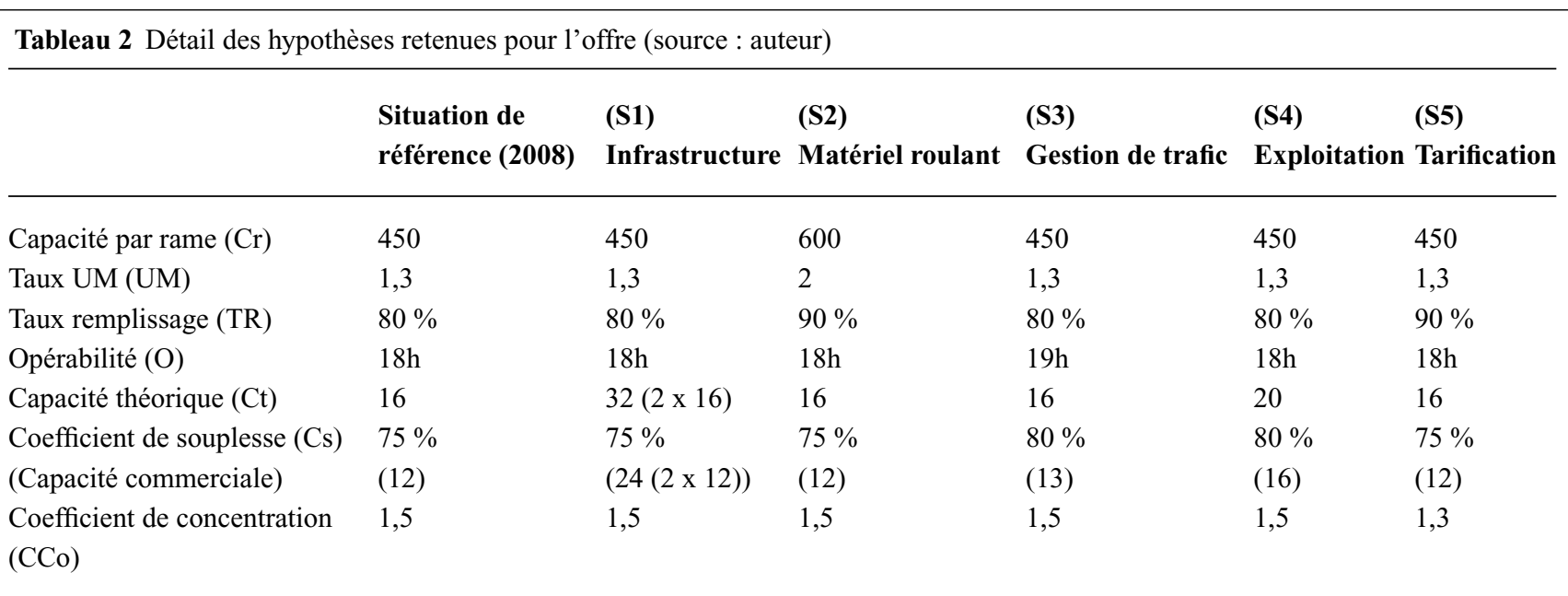


en vigueur sur la ligne (TVM 300) pour une résilience en heure de pointe de $75 \%$ de la capacité théorique. La capacité commerciale obtenue est donc de 12 trains par heure.

Enfin, le coefficient de concentration est obtenu par comparaison de la densité de trafic entre la période de pointe et la période creuse. L'hypothèse est faite que ce différentiel peut se transcrire du nombre de trains au nombre de voyageurs en tenant compte du fait qu'il ne peut y avoir sur-remplissage des TGV, la réservation étant obligatoire. Le différentiel obtenu est de 1,5 .

\section{Résultats et analyse par scénario}

\section{Situation de référence (2008)}

Si on rapporte les perspectives de demande aux conditions de capacité en 2008, le risque de saturation de l'infrastructure en capacité commerciale est identifié à l'horizon 2020-2025.

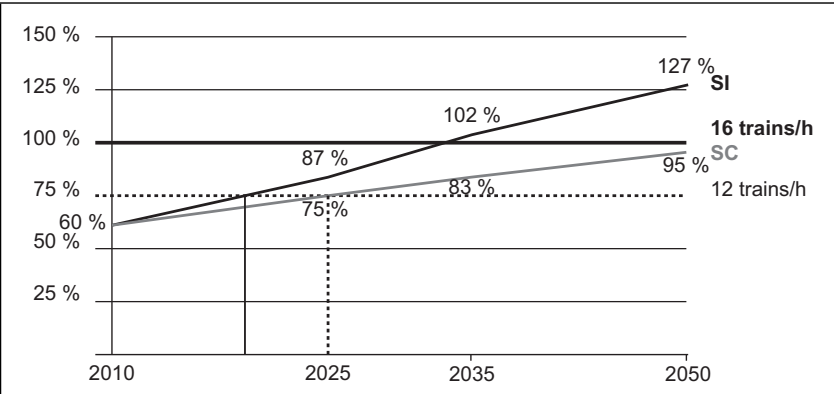

Fig. 3 Taux d'utilisation de la LGV à l'horizon 2050 selon situation observée sur le réseau en 2008 (Source : auteur)

Le Scénario de demande corrigé (SC) montre un horizon de saturation repoussé de cinq années par rapport au Scénario initial (SI). La demande est en effet inférieure de 8 millions de voyageurs en 2025 et 21 millions en 2050 . Cette différence tient pour $80 \%$ en 2025 à l'effet réseau. Elle est ensuite amplifiée par le différentiel de croissance retenu mais surtout par l'hypothèse d'un découplage entre la vitesse et le PIB. Par conséquent, alors que le scénario initial prévoit un doublement des trafics à l'horizon 2050, le scénario corrigé prévoit une augmentation plus modeste de $60 \%$ de la demande.

Pour retarder le phénomène de saturation sans modifier les conditions de production, il est possible de relever la limite de la capacité commerciale pour libérer plus de capacité et repousser l'horizon de saturation. Néanmoins, cette action se traduirait par un accroissement du risque d'irrégularité et par une augmentation forte du risque de retard.

Enfin, on observe que le taux d'utilisation de la capacité théorique est de $60 \%$ en 2008 d'après la méthode. Ce résultat correspond aux observations réalisées sur le terrain (moyenne de 10 trains par heure aux heures de pointe).

\section{Scénario S1 : dédoublement de l'infrastructure}

Le dédoublement de l'infrastructure est le scénario privilégié par les acteurs ferroviaires (gestionnaire d'infrastructure et opérateur historique). La solution envisagée est la réalisation d'une ligne nouvelle entre Paris-Orléans-Clermont-Ferrand-Lyon pour un coût de 14 Mds $\subset$ [9].

Le gain attendu en capacité commerciale est de $100 \%$ à conditions d'exploitation, de gestion et d'offre commerciale identiques. L'horizon de saturation est reporté au-delà de 2050 pour une mise en service à horizon 2025.

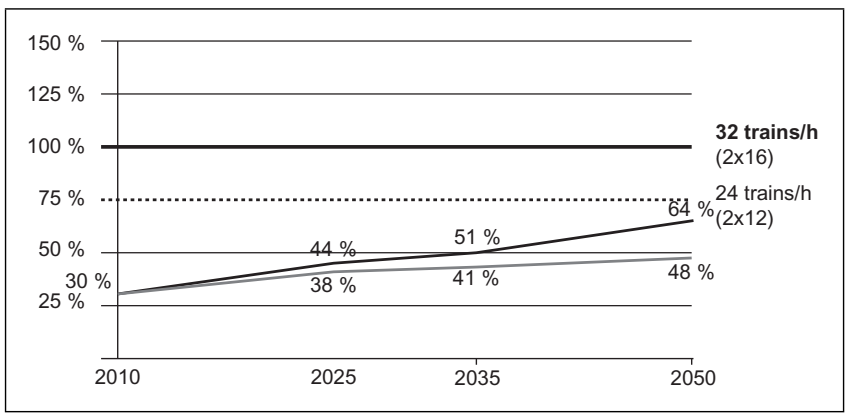

Fig. 4 Taux d'utilisation de la LGV à l'horizon 2050 selon le scénario S1 (Source : auteur)

Outre son coût élevé, ce scénario présente le risque d'un report de la saturation de la ligne ferroviaire vers les nœuds ferroviaires. Il est donc fortement probable que des aménagements lourds soient à réaliser en annexe $\mathrm{du}$ projet dans les principales gares d'interconnexion. Ce coût induit peut se révéler important notamment sous la contrainte urbanistique (création d'un tunnel à Lyon Part-Dieu ?).

\section{Scénario S2 : le TGV « haute densité »}

Une piste d'évolution considérée pour le matériel roulant est la définition de TGV à « haute densité » [38]. Ce scénario fait l'hypothèse de la diffusion d'une solution de type Ouigo (TGV low cost) à l'ensemble de l'offre commerciale active sur la ligne Paris-Lyon à horizon 2025. La capacité théorique moyenne des rames est portée à 600 places. Cette hypothèse prend en compte l'évolution des rames Duplex de 512 places vers 634 places et le remplacement des rames réseau (377 places) par des rames de type AGV 10 de 462 places. L'augmentation en capacité des rames Duplex est essentiellement obtenue par la diffusion du standard seconde classe à l'ensemble des voitures. Le taux de rame 
double est porté à sous l'effet d'une optimisation de la gestion du matériel roulant et le taux de remplissage est optimisé grâce à une plus grande discrimination tarifaire et une optimisation de la gestion de la vente et des réservations de billets.

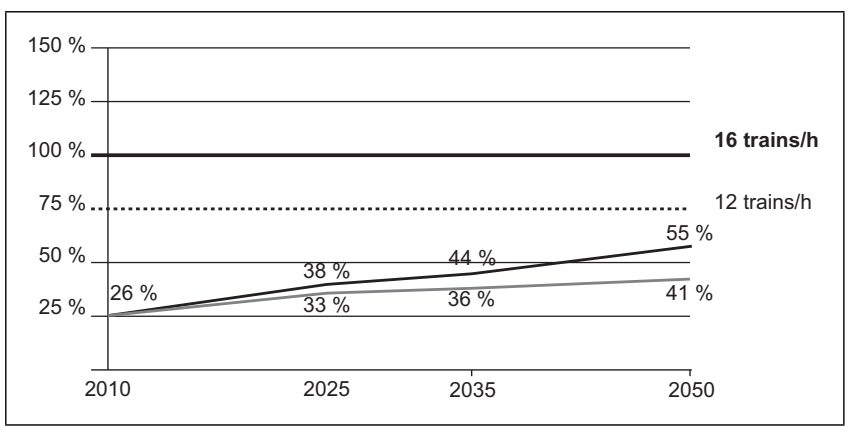

Fig. 5 Taux d'utilisation de la LGV à l'horizon 2050 selon le scénario S2 (Source : auteur)

Ce scénario permet un gain en capacité de $131 \%$ par rapport à la situation en 2008. Il repousse l'horizon de saturation au-delà de 2050. Ce résultat est obtenu sans modification des conditions d'exploitation et de gestion.

Le coût lié à cette migration du matériel est a priori réduit et peut s'inscrire dans le cycle de vie du matériel. Les rames Duplex mises en service en 1997 devront être réhabilitées progressivement tandis que les rames réseaux en circulation depuis les débuts du TGV (1981) devraient être renouvelées.

Néanmoins, si ce scénario apparaît crédible d'un point de vue technique, il interroge sur la capacité à évoluer du modèle économique jusque-là fondé sur la première classe et l'espacement confortable des sièges. Dans ce cadre, l'évolution de l'offre vers le standard seconde classe peut induire des problèmes d'acceptation sociale et de rentabilité économique. Plus généralement, c'est la conception de la grande vitesse et des services associés qui est remise en cause par ce scénario : quelle évolution du service rendu à l'usager et quelle tarification pour compenser la « perte » de confort?

Pour finir, si le résultat est tout aussi intéressant que le doublement de l'infrastructure, il doit être relativisé en cas de concurrence. En effet, le raisonnement est produit selon une situation de monopole. Il n'est pas certain que l'effet de politiques commerciales de différents opérateurs en concurrence puisse être le même. Cela reste à tester selon les conditions d'ouverture à la concurrence (franchise, open access, etc.).

\section{Scénario S3 : optimiser la gestion des flux}

Ce scénario teste une adaptation à la marge la capacité quotidienne. C'est une solution de court terme mais qui peut se révéler utile dans la gestion de la demande.

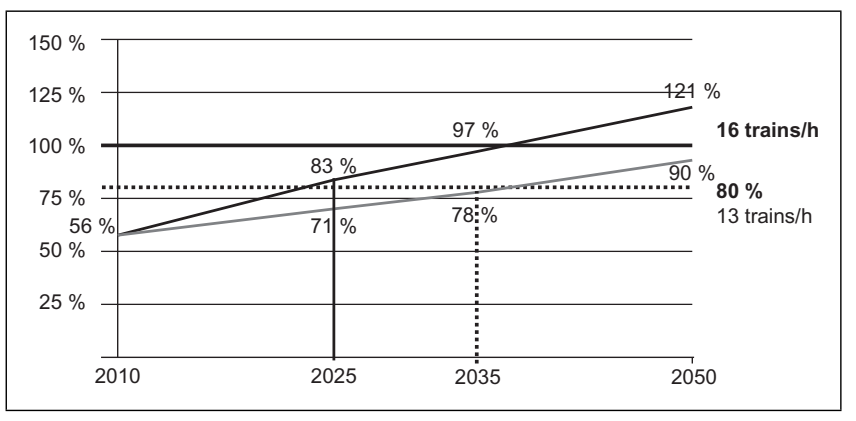

Fig. 6 Taux d'utilisation de la LGV à l'horizon 2050 selon le scénario S3 (Source : auteur)

Le scénario s'inspire de la nouvelle grille horaire définie par RFF en 2013 suite à la mise en service de la LGV Rhin-Rhône [39]. La capacité commerciale est portée à $80 \%$ de la capacité théorique un jour par semaine (vendredi soir) et pour une heure (13 trains). L'hypothèse retenue est une généralisation de cette solution à l'ensemble des heures de pointe.

Le gain en capacité est de $13 \%$ et l'horizon de saturation est reporté de 5 à 15 ans. Ce résultat peut être obtenu par une meilleure robustesse de la grille de circulation, une meilleure gestion du flux TGV en temps réel et par un meilleur respect des règles de conduite de la part des conducteurs.

Néanmoins, ce résultat induit une dégradation potentielle de la qualité de service. Le relèvement de la capacité commerciale sans évolution réelle des méthodes de production peut accroitre le risque de retard ( $c f$. section 2) et dégrader la régularité des circulations. Par conséquent, il nécessite sans doute une meilleure prise en compte du phénomène de congestion à la fois par le gestionnaire d'infrastructure mais aussi par l'opérateur.

Il est donc important de définir ici ce que l'on attend en termes de fiabilité du service pour fixer le coefficient de souplesse optimal.

\section{Scénario S4 : améliorer l'exploitation}

Le scénario exploitation teste l'effet d'un relèvement de la capacité théorique par l'introduction d'une innovation de rupture. Cette innovation consiste à implémenter sur la ligne le nouveau système de signalisation européen ERTMS niveau 2 en lieu et place du système existant (TVM 300). Ce système est réputé permettre une gestion plus fine des circulations en réduisant l'intervalle entre chaque circulation de 4 min à $3 \mathrm{~min}$, c.-à-d. 20 trains par heure en théorie pour 16 trains par heure en pratique $[39,40]$. Le coefficient de souplesse est aussi amélioré ( $80 \%$ ).

Le gain en capacité est de $33 \%$ et l'horizon de saturation est reporté de 15 à 25 ans. Ce scénario permet une plus 


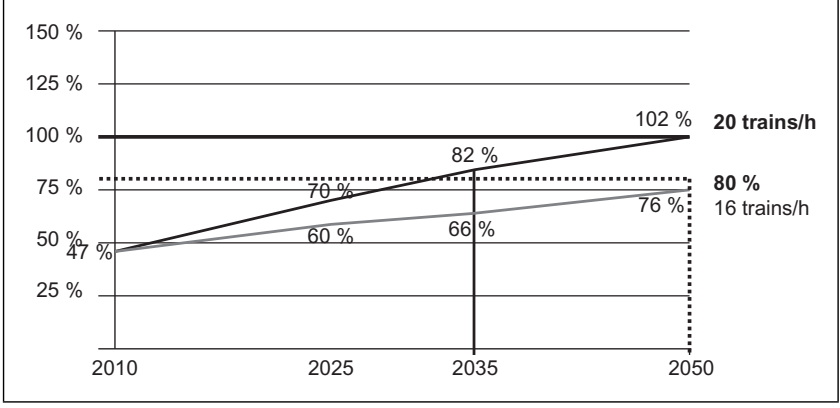

Fig. 7 Taux d'utilisation de la LGV à l'horizon 2050 selon le scénario S4 (Source : auteur)

grande capacité sur la ligne sans optimisation particulière de l'offre de la part de l'opérateur.

Néanmoins, il nécessite une coordination des investissements. Le système de signalisation installé sur les voies doit correspondre au système de signalisation en cabine pour être opérationnel. Le choix du gestionnaire d'infrastructure doit donc être suivi par l'opérateur. Or il a été montré qu'il peut exister de nombreuses barrières à la migration dans le cas où l'opérateur historique ne s'aligne pas sur le choix du gestionnaire d'infrastructure [41]. On observe que c'est en général sur les lignes les plus ouvertes à la concurrence que la migration est la plus réussie sur le modèle de la Betuwe line aux Pays-Bas ou des tunnels suisses [41]. Le coût estimé de ce scénario est de 500 millions d'euros pour la LGV Paris-Lyon [42]. La répartition est égale entre le gestionnaire et l'opérateur dominant pour lequel la migration du matériel roulant peut être réalisée progressivement selon le principe du cycle de vie des rames.

\section{Scénario S5 : Économie de la congestion ferroviaire}

Enfin, le scénario tarification adopte le principe d'une économie de la congestion ferroviaire [4] défendue par RFF dans sa proposition de tarification de la congestion en 2012. La saturation de la capacité commerciale en heure de pointe entraîne une augmentation du prix du sillon (tarification de la congestion) qui se traduit par une réduction du coefficient de concentration $(1,3)$. Cette évolution «idéale » pour le gestionnaire d'infrastructure produit une meilleure répartition des circulations entre heure de pointe et heure creuse. Le taux choisi est inspiré du modèle japonais où le différentiel en matière d'offre entre heure de pointe et heure creuse est de 1,3 sur la ligne Tokyo-Osaka en 2013. Côté opérateur, l'hypothèse est faite que le signal prix émis par le gestionnaire d'infrastructure est répercuté dans la politique tarifaire (effet rareté) qui se traduit en heure de pointe par un taux de remplissage optimisé $(90 \%)$ sans évolution particulière de l'offre en capacité (paramètres inchangés). Pour autant, l'hypothèse d'une bonne transmission du signal prix entre le gestionnaire d'infrastructure et le voyageur reste à vérifier.

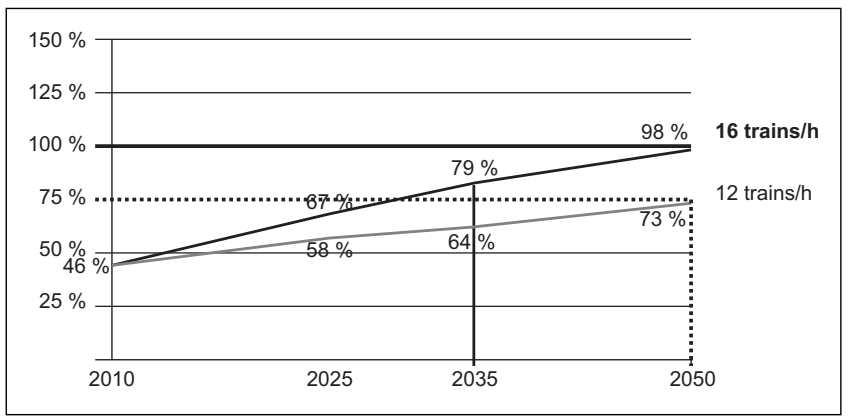

Fig. 8 Taux d'utilisation de la LGV à l'horizon 2050 selon le scénario S5 (Source : auteur)

Le gain en termes de capacité est d'environ $30 \%$ et l'horizon de saturation est reporté de 10 à 25 ans. Le coût représenté par un tel scénario peut se traduire par un gain financier en optimisant la valeur d'usage du réseau (maximisation des surplus).

Cependant, il pose un vrai problème en matière d'acceptabilité sociale (désheurage, discrimination tarifaire) et peut s'avérer être négatif pour la collectivité dans le cas où le gestionnaire ne réinvestirait pas le produit des surplus dans des projets capacitaires.

Il est intéressant de noter que la réalisation d'un tel scénario serait aujourd'hui très certainement limitée par la régulation tarifaire du prix des billets contrôlée par l'État [43] et par le développement de la concurrence intermodale.

\section{Discussion : scénario « d'optimisation générale »}

En définitive, deux options se distinguent face au problème de saturation. La création d'une nouvelle infrastructure au prix d'un lourd investissement ou l'évolution des outils et méthodes de production dans une logique de rendements croissants.

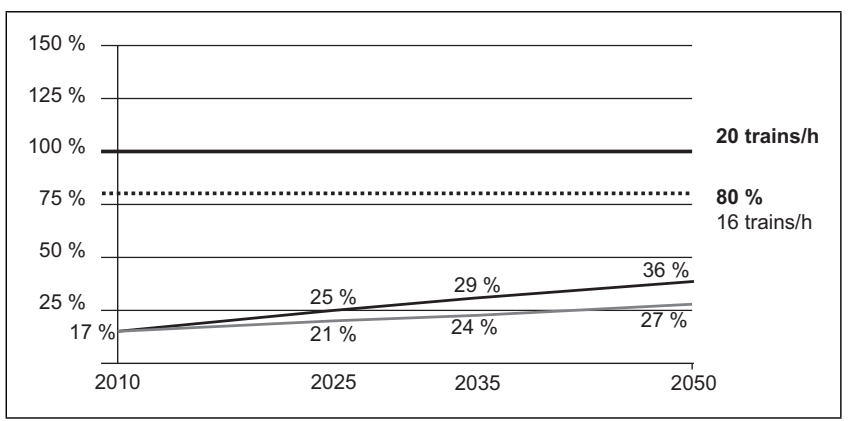

Fig. 9 Taux d'utilisation de la LGV à l'horizon 2050 selon un scénario «d'optimisation générale » (Source : auteur) 
Tableau 3 Synthèse des résultats obtenus pour chacune des scénarios (Source : auteur)

\begin{tabular}{lllll}
\hline Scénario & Gain en capacité & Coût scénario & Horizon saturation & Temporalité mise en ouvre \\
\hline (S1) matériel roulant & $+131 \%$ & Cycle de vie du matériel & Post 2050 & Moyen terme \\
(S2) Infrastructure & $+100 \%$ & $13 \mathrm{Mds}$ C & Post 2050 & Long terme \\
(S3) Gestion de trafic & $+13 \%$ & Nul & $2025-2040$ & Court terme \\
(S4) Exploitation & $+33 \%$ & $500 \mathrm{MC}$ & $2035-2050$ & Moyen terme \\
(S5) Tarification & $+30 \%$ & Nul & $2030-2050$ & Court terme \\
(S6) Optimisation générale & $+200 \%$ & $500 \mathrm{MC} /$ cycle de vie des rames & Post 2050 & Moyen terme \\
\hline
\end{tabular}

Ce dernier scénario, synthèse des scénarios précédents, fait l'hypothèse d'une évolution globale de l'activité sous contrainte de l'infrastructure existante. Il suppose l'implémentation de l'ERTMS, une tarification de la congestion, la mise en service de $\mathrm{TGV}$ « haute densité » et l'optimisation de la gestion des trains.

Le gain en capacité lié à ce scénario «d'optimisation générale » permettrait de plus que doubler la capacité existante. Son coût peut être optimisé notamment en réduisant l'impact de l'immobilisation des rames grâce à une mutualisation entre leur migration vers ERTMS et leur réaménagement vers plus de capacité.

Néanmoins, il présente la particularité de concentrer l'ensemble des risques et obstacles liés à l'évolution des outils et méthodes de production. Il suppose en effet pour l'opérateur de repenser l'offre commerciale en grande vitesse, d'intégrer de nouvelles conditions d'exploitation (ERTMS) et pour le gestionnaire d'infrastructure de trouver le bon signal prix pour favoriser l'étalement de la pointe.

\section{Conclusion}

Ce travail met donc en perspective la relativité du concept de saturation d'une ligne ferroviaire. Le concept varie d'une part selon le type de capacité envisagé (théorique ou commerciale) et d'autre part selon les choix réalisés par les acteurs en termes de performance et de qualité de service.

Les résultats obtenus pour la LGV Paris-Lyon montrent qu'il est nécessaire pour le système ferroviaire français d'apprendre à gérer l'arrivée à maturité des LGV. Trente ans après sa mise en circulation, la LGV Paris-Lyon est la première à poser le problème de saturation selon les conditions d'exploitation, d'offre commerciale et de gestion définies initialement. On peut supposer que les LGV Paris-Tours et Paris-Lille arriveront également d'ici quelques années à un niveau d'utilisation proche de la saturation de la capacité commerciale. Le cas de la LGV Paris-Lyon peut donc faire office de laboratoire d'expérimentation pour l'avenir du réseau LGV à condition que l'on accepte de modifier les règles du jeu initiales vers un service TGV plus massifié et un usage aujourd'hui banalisé.

Ainsi, trois remarques conclusives peuvent être retenues. Dans un premier temps, on montre qu'il existe une réserve en capacité importante sur la ligne Paris-Lyon à condition d'évoluer dans la conception de l'offre commerciale et de l'exploitation. L'intégration des nouvelles technologies et de nouvelles méthodes de management de la capacité peuvent être une réponse pertinente. En second lieu, on interpelle sur la nécessité de prendre en compte le risque de saturation des gares. L'étude de la capacité d'une gare pourrait constituer une suite logique à ce travail. Enfin, on pose directement la question du lien entre gestion de la capacité et régulation du système ferroviaire : quelle régulation adopter pour inciter les acteurs à la mise en œuvre de solutions innovantes et assurer une utilisation optimale de la capacité ferroviaire?

\section{Bibliographie}

1. DGITM (2011). Schéma national des infrastructures de transport. Avant-projet consolidé, Paris.

2. Auphan E (2012). Le TGV ou le démantèlement du réseau ferré français. Annales de géographie $684:$ 194-213.

3. Dumont F (2012). La fin du tout TGV. Ville Rail \& Transport 535 : 26-35.

4. Brunel J, Marlot G, Perez M (2013). Measuring congestion in rail sector: the French experience. 13th WCTR, 15-18 juillet, Rio de Janeiro.

5. Abril M, F. Barber F, Ingolotti L, Salido MA, Tormos P, Lova A (2008). An assessment of railway capacity. Transportation Research Part E 44 : 774-806.

6. RFF (2011). Perspectives de trafic et de circulation Sud-Est : LGV Paris-Lyon et LGV POCL. RFF, Paris.

7. LET-ENERDATA (2012). Recherche : Enjeux spatiaux, économiques et politiques des scénarios de mobilité durable à l'horizon 2050. Rapport de la tâche 7 : spatialisation des flux, Lyon.

8. Schafer A (2001). Long-Term Trends in Global Passenger Mobility. National Academy of Engineering Website, www.nae.edu/.

9. Commission Mobilité 21 (2013). Mobilité 21: «pour un schéma national de mobilité durable ». Rapport, Paris. 
10. Luzeaux D, Ruault JR (2008). Ingénierie des systèmes de systèmes : méthodes et outils. Hermes Science Publications, Paris.

11. Lai YC (2008). Increasing railway efficiency and capacity through improved operations, control and planning. Ph.D. Dissertation, University of Illinois at Urbana-Champaign.

12. Martland CD, Hutt G (2005). Analysis of potential approaches to interline capacity flow management, RaiLinc, Cary.

13. Petersen ER (1974). Over the road transit time for a single track railway. Transportation Science 8: 65-74.

14. Higgins A, Ferreira Kozan E (1996). Optimal scheduling of train on a single track. Transportation research part B: methodological 30: $147-161$.

15. Kraft ER (1987). A branch and bound procedure for optimal trains dispatching. Journal of the transportation research forum 28 : 263-276.

16. Higgins A, Kozan E, Ferreira L (1995). Modelling delay risks associated with train schedules. Transportation Planning and Technology 19 : 89-108.

17. Dingler M, Barkan C, Koenig A, Sogin S. (2010). Determining the causes of train delay. 2010 Annual AREMA Conferences, 29 august, Orlando.

18. Kraft E.R. (1982). Jam capacity of single track rail lines. Proceedings of the Transportation Research Forum 23 : 461-471.

19. Burdett RL, Kozan E (2006). Techniques for absolute capacity determination in railways. Transportation Research Part B 40: 616-632.

20. Liotta G, Cicini P, De Luca P (2009). A simulation-based approach for estimating the commercial capacity of railways. In : Proceedings of the 2009 Winter Simulation Conference, Washington.

21. Caralp R (1951). L'évolution de l'exploitation ferroviaire en France. Annales de géographie 322 : 321-336.

22. Krueger H (1999). Parametric modeling in rail capacity planning. In : Proceedings of the 1999 Winter Simulation Conference, Washington.

23. Harrod S (2007). Rail capacity and management planning. Ph.D. Dissertation, University of Cincinnati.

24. Lai YC, Barkan C (2009). An enhanced parametric railway capacity evaluation tool (RCET). Transportation Research Record $2117: 33-40$.

25. Harker PT, Hong S (1994). Pricing of track time in railroad operations: An internal market approach. Transportation Research Part B 28 : 197-212.

26. Ferreira L (1997). Rail track infrastructure ownership: investment and operational issues. Transportation 24 : 183-200.
27. Nilsson JE (2002). Towards a welfare enhancing process to manage railway infrastructure access. Transportation Research Part A 36 : 419-436.

28. Gibson S (2003). Allocation of capacity in the rail industry. Utilities Policy 11 : 39-42.

29. Nash CA, Matthews B (2005). Rail infrastructure charges-the issue of scarcity. Working paper, University of Leeds.

30. Quinet E (2003). Short term adjustments in rail activity: the limited role of infrastructure charges. Transport Policy 10:73-79.

31. Johnson D, Nash C (2008). Charging for scarce rail capacity in Britain: A case study. Review of Network Economics 7.

32. Landex A, Kaas AH (2005). Planning the most suitable travel speed for high frequency railway lines. In : the $1 s t$ International Seminar on Railway Operations Modelling and Analysis, Netherlands.

33. Harrod S (2009). Capacity factors of a mixed speed railway network. Transportation Research Part E 45 : 830-841.

34. SETRA (2009). Le transport ferroviaire de voyageurs sur le réseau ferré national français. Synthèse des connaissances, Bagneux.

35. Gibson S, Cooper G, Ball B (2002). The evolution of capacity charges on the UK rail network. Journal of Transport Economics and Policy 36 : $341-354$.

36. Cambridge Systematics (2007). National rail freight infrastructure capacity and investment study. Association of American Railroads, Cambridge.

37. Becker J-J, Delache X, Brunel J, Sigaud D, Sauvant A (2013). Estimation des élasticités des trafics routiers et ferroviaires au PIB. In rapport Quinet, Evaluation socio-économique en période de transition, Tome 2, Rapports et documents, Commissariat général à la stratégie et à la prospective, Paris.

38. Charlier L (2013). TGV Ouigo : les deux trains réaliseront un tour de la Terre chaque semaine. La lettre ferroviaire $82: 1-3$.

39. Delaborde F (2012). Augmentation de capacité de la LGV ParisLyon. Le Rail 185 : 30-35.

40. Lacôte F (2012). Quelle performance attendue de l'ERTMS ? Le Rail $185: 36$

41. Guihéry L, Laroche F (2013). European Rail Traffic Management System (ERTMS): Supporting competition on the European rail network ? Research in Transportation Business \& Management $6: 81-87$.

42. Leboeuf M (2014). Grande vitesse ferroviaire. Collection Documents, Paris.

43. Perennes $\mathrm{P}$ (2012). Pourquoi les tarifs de la SNCF sont-ils régulés? Concurrence 3. 